\section{P27 YOUTH PERSPECTIVES ON SOFT DRINKS AFTER THE INTRODUCTION OF THE UK SOFT DRINKS INDUSTRY LEVY: A FOCUS GROUP STUDY USING REFLEXIVE THEMATIC ANALYSIS}

${ }^{1}$ Catrin Penn-Jones*, ${ }^{2}$ Tarra Penney, ${ }^{1}$ Roxanne Armstrong-Moore, ${ }^{3}$ Steven Cummins, ${ }^{1}$ Martin White. ${ }^{1}$ Centre for Diet and Activity Research, MRC Epidemiology Unit, University of Cambridge, Cambridge, UK; ${ }^{2}$ School of Global Health, York University, Toronto, Canada; ${ }^{3}$ Department of Public Health, Environments and Society, London School of Hygiene and Tropical Medicine, London, UK

\subsection{6/jech-2021-SSMabstracts.116}

Background Soft drinks consumption is a key target area of the UK Childhood Obesity strategy, with $36 \%$ of young people reporting consuming sugary drinks 2-4 times per week. The Soft Drinks Industry Levy (SDIL) was implemented in the UK in April 2018 to help tackle this issue and is designed to incentivise the reformulation of eligible soft drinks. To date, no study has explored youth perspectives of the SDIL through primary qualitative research. Therefore, this study aimed to explore perceptions of sugary drinks and the SDIL among adolescents in the UK, following its implementation on 6th April 2018, to investigate perspectives on this policy from a key target group.

Methods Four focus groups were conducted between December 2018 - May 2019 with 23 participants aged 11-14 years from schools in Essex, UK. A semi-structured topic guide aimed to elicit perspectives on sugar, sugary drinks and the SDIL. We also included a group task to rank a selection of UK soft drinks based on their sugar content, aiming to elicit engaged discussion. Braun and Clarke's reflexive thematic analysis was used to analyse the data inductively.

Results Four main themes were found during analysis: 1) Sugary drinks are bad for you, but young people still consume them, 2) Mixed awareness of and ambivalence towards the SDIL, 3) Marketing influences the perceptions of drinks, regardless of SDIL, and 4) Suggestions for additional, low agency interventions to reduce sugar consumption.

Conclusion Participants demonstrated high awareness that sugary drinks were detrimental to their health, however, this sometimes did not translate into holding positive views of the SDIL - a fiscal policy designed to reduce sugar in drinks. Marketing was a strong influence on consumption and the perception of drinks, sometimes unconsciously and independently of the SDIL. Interventions where participants had to do little to reduce their sugar consumption were preferred, implying that future policies should be constructed that require little engagement from young people to reduce their sugar consumption. High agency education-based interventions may be less appropriate, as participants already understand the negative consequences of excess sugar and SSB consumption.

\section{P28 SOCIAL RELATIONSHIPS AND COGNITIVE DECLINE IN ADULTHOOD: AN INVESTIGATION FROM THE MRC NATIONAL SURVEY OF HEALTH AND DEVELOPMENT AND THE ENGLISH LONGITUDINAL STUDY OF AGEING}

\footnotetext{
${ }^{1}$ Jane Maddock* , ${ }^{2}$ Anna Marseglia, ${ }^{2}$ Federico Gallo, ${ }^{2}$ Serhiy Dekhtyar, ${ }^{2}$ Anna-Karin Welmer, ${ }^{3}$ Frank J Wolters, ${ }^{3}$ Mohammad Arfan Ikram, ${ }^{1}$ Daniel Davis, ${ }^{1}$ George B Ploubidis, ${ }^{1}$ Marcus Richards. 'MRC Unit for Lifelong Health and Ageing at UCL, UCL, London, UK; ${ }^{2}$ Aging Research Center, Karolinska Institutet, Stockholm, Sweden; ${ }^{3}$ Department of Epidemiology, Erasmus MC, Rotterdam, Netherlands
}

Background It is difficult to determine the nature of the association between social relationships(SR) and cognitive capability, as different aspects of the former likely have different effects on the latter. Moreover, whether SRs are associated solely with cognitive performance or also with rate of decline remains unclear. We aim to examine associations between structural (marital/cohabitation status, network size) and interactional (frequency of contact, social participation, positive and negative social support) aspects of SR with average and change in measures of memory, executive function and processing speed over time.

Methods We used data from the MRC National Survey for Health and Development (NSHD, $n=2,109)$ and the English Longitudinal Study of Ageing(ELSA, $n=8460)$. Participants from NSHD aged $53 \mathrm{y}$ at baseline were followed up three times over 16y. Participants from ELSA aged 64.3y(9.8SD) at baseline were followed up 9 times over $17 y$. We applied multilevel models to examine each SR variable at baseline with each standardised measure of cognitive performance. We first examined average performance adjusting for time, then rate of decline by including an interaction term between SR and time. Models were adjusted for age at baseline, sex, social class, education, limitations in activities of daily living, and health conditions. We adjusted for depressive symptoms in sensitivity analyses.

Results In NSHD, having more positive support or less negative support was associated with higher memory scores ( $\beta: 0.05$ [95\%CI:0.02-0.09]; $\beta 0.06[95 \% \mathrm{CI}: 0.02-0.09]$ ), but not processing speed(executive function not measured). We did not observe associations between the other measures of SR and cognitive performance in NSHD. In ELSA, being married ( $\beta: 0.04$ [95\%CI:0.01-0.08]), having a larger network size ( $\beta: 0.12[95 \%$ CI:0.02-0.21] $>6$ vs. zero), more frequent contact ( $\beta: 0.25$ [95\%CI:0.08-0.24] $1-2 /$ week vs <1/year), greater social participation ( $\beta: 0.31[95 \% \mathrm{CI}: 0.28-0.35]$ high vs low), more positive support ( $\beta: 0.02[95 \% \mathrm{CI}: 0.01-0.03])$, or less negative support ( $\beta: 0.04[95 \% \mathrm{CI}: 0.02-0.05])$ was associated with higher memory scores. Similar associations were observed for executive function but not processing speed. We did not observe any associations between SRs and decline in memory or processing speed in NSHD. In ELSA, being married and greater social participation were associated with less decline in memory and executive function but not processing speed.

Discussion Preliminary results suggest a relationship between both structural and interactional aspects of SRs with memory, and executive function but not processing speed. However, only marital status and social participation were associated with decline in memory and executive function over time in one cohort. We will also present replication of these analyses in the population-based SNAC-K and Rotterdam Study cohorts.

\section{P29 COMMON HEALTHCARE VISITS PRIOR TO THE DIAGNOSIS OF DEMENTIA}

\footnotetext{
${ }^{1,2}$ TienYu Owen Yang ${ }^{*},{ }^{3}$ Yen-Chen Wang, ${ }^{4}$ Yun-Cheng Zhang, ${ }^{4}$ Yi-Fang Chuang. ${ }^{1}$ Nuffield Department of Population Health, University of Oxford, Oxford, UK; ${ }^{2}$ University Hospitals Coventry and Warwickshire, Coventry, UK; ${ }^{3}$ Faculty of Medicine, National Yang Ming Chiao Tung University, Taipei City, Taiwan; ${ }^{4}$ Institute of Public Health, National Yang Ming Chiao Tung University, Taipei City, Taiwan
} 